\title{
Testing Disk Instability Models for Giant Planet Formation
}

\author{
Alan P. Boss \\ Department of Terrestrial Magnetism, Carnegie Institution of Washington, 5241 Broad \\ Branch Road, NW, Washington, DC 20015-1305 \\ boss@dtm.ciw.edu
}

\begin{abstract}
Disk instability is an attractive yet controversial means for the rapid formation of giant planets in our solar system and elsewhere. Recent concerns regarding the first adiabatic exponent of molecular hydrogen gas are addressed and shown not to lead to spurious clump formation in the author's disk instability models. A number of disk instability models have been calculated in order to further test the robustness of the mechanism, exploring the effects of changing the pressure equation of state, the vertical temperature profile, and other parameters affecting the temperature distribution. Possible reasons for differences in results obtained by other workers are discussed. Disk instability remains as a plausible formation mechanism for giant planets.
\end{abstract}

Subject headings: solar system: formation - planetary systems

\section{Introduction}

The disk instability mechanism for giant planet formation is based on the formation and survival of self-gravitating clumps of gas and dust in a marginally gravitationally unstable protoplanetary disk (Boss 1997; reviewed by Durisen et al. 2007). In order for a disk instability to succeed, the disk must be able to cool its midplane as the clumps form, allowing them to continue to contract to higher densities, and the clumps must be able to survive indefinitely in the face of Keplerian shear, tidal forces, and internal thermal pressure. Considering the complicated physical processes involved during the time evolution of a three dimensional disk instability, it is perhaps not surprising that the theoretical basis for the disk instability hypothesis remains unclear even after a decade of work on the subject.

The Indiana University (IU) group has been active in studying disk instabilities, and has generally found that disk instabilities are unable to lead to the formation of self-gravitating, 
dense clumps that could go on to form gas giant protoplanets (e.g., Pickett et al. 2000; Mejía 2004; Cai et al. 2006a,b; Boley et al. 2006, 2007a,b). On the other hand, the Washington-Zurich group (e.g., Mayer et al. 2002, 2004, 2007) has presented models that support the hypothesis that disk instabilities can lead to the formation of long-lived giant gaseous protoplanets. We present here several new calculations that attempt to understand the reasons for some of these different outcomes regarding disk instability.

\section{Energy Equation of State}

Boley et al. (2007a) pointed out that uncertainties about the ortho/para ratio of molecular hydrogen at low temperatures might lead to differences in the outcome of disk instability models. They suggested that a mixture intermediate between pure parahydrogen and a 3:1 ortho/para ratio might be the most appropriate choice. Boley et al. (2007a) also noted that discontinuities in the specific internal energy equation for molecular hydrogen could lead to artificially low values of the first adiabatic exponent for a simple perfect gas ( $\Gamma_{1}=\gamma=1+R_{g} /\left(\mu c_{V}\right)$, where $R_{g}$ is the gas constant, $\mu$ is the mean molecular weight, and $c_{V}$ is the specific heat at constant volume; Cox \& Giuli 1968). Values of $\gamma \leq 4 / 3$ can lead to dynamical instabilities (either expansion or contraction) away from a configuration of hydrostatic equilibrium (Cox \& Giuli 1968). Boley et al. (2007a) suggested that $\gamma \leq 4 / 3$ could artificially lead to clump formation.

Boley et al. (2007a) stated that the energy equation of state (EOS) used in disk instability models by Boss $(2001,2002 b, 2005)$ contained a discontinuity that might be responsible for artificially lowering $\gamma$ below 4/3, leading to spurious fragmentation. Boley et al. (2007a) based this assertion on the equations of state described by Boss (1984). However, all of the hydrodynamical models run by the present author since Boss (1989) have been based on a different energy equation than that reported by Boss (1984), as a result of a direct comparison with the equation of state routines employed by Werner Tscharnuter. The models of Boss (1989) revised the energy equation treatment to include an interpolation between temperatures of $100 \mathrm{~K}$ and $200 \mathrm{~K}$, but this revision was not explicitly stated in the Boss (1989) paper as it seemed insignificant at the time.

Using the notation of Boss (1984), the specific internal energy for molecular hydrogen for temperatures $T<100 \mathrm{~K}$ is taken to be $E_{H_{2}}^{*}=3 / 2 R_{g} T / \mu$, while for temperatures between $100 \mathrm{~K}$ and $600 \mathrm{~K}$ it is $E_{H_{2}}^{*}=5 / 2 R_{g} T / \mu$. For intermediate temperatures, $100 \mathrm{~K}<\mathrm{T}<200 \mathrm{~K}$, the internal energy is interpolated according to the equation $E_{H_{2}}^{*}=3 / 2 R_{g} T / \mu[1+2 / 3(T-$ 100)/100]. The Erratum by Boley et al. (2007b) used this revised EOS. 
Boley et al. (2007a,b) showed that for a pure parahydrogen or 3:1 ortho:para mix, $\gamma$ decreases significantly at $\sim 100 K$, but does not fall below $4 / 3$. Figure 1 depicts the behavior of $\gamma$ calculated with the revised Boss equation of state and shows that $\gamma$ drops below $4 / 3$ for $135 K<T<200 K$. The densest clumps found in Boss's disk instability models generally have maximum temperatures below $135 K$, and even lower mean temperatures. Boss (2005) presented a disk instability model with the highest spatial resolution computed to date, and found that the densest clump that formed had a maximum temperature of $120 \mathrm{~K}$ and a mean temperature of $94 K$. Boss (2006c) found that clumps formed in disk instabilities around M dwarf protostars had maximum temperatures less than 100K. Boss's (2002a) Table 1 showed maximum clump temperatures of $115 \mathrm{~K}$ to $126 \mathrm{~K}$ for a range of models with varied opacities.

Perhaps the most important point raised by Boley et al. (2007a, b) is that $\gamma$ is likely to decrease significantly around $100 K$, and this softening of the pressure EOS will enhance the formation of dense clumps, as happens in locally isothermal disks with an effective $\gamma=1$. In fact, Figure 1 in Boley et al. (2007b) shows that the Boss $\gamma$ is higher than that of either of the preferred hydrogen mixtures for $T<100 K$, implying that clump formation is suppressed somewhat in the Boss models as a result.

\section{Pressure Equation of State}

Several new models have explored using the same pressure EOS as is used in the IU group models (e.g., Cai et al. 2006a,b; Boley et al. 2006): the gas pressure $p$ is given by $(\gamma-1) \rho E$, where $\gamma=5 / 3, \rho$ is the gas density, and $E$ is the specific internal energy of the gas. Three models were run with this pressure EOS, starting from the same initial disk model as model HR in Boss (2001) - a 0.091 $M_{\odot}$ disk orbiting a $1 M_{\odot}$ protostar with an outer disk radius of $20 \mathrm{AU}$. The calculations were made with the same three dimensional, gravitational, radiative hydrodynamics code as in Boss (2001) and in all subsequent Boss disk instability calculations (see these earlier papers for more details about the calculational techniques and initial conditions). The models had $N_{\phi}=256$ and $N_{Y l m}=32$, though, compared to $N_{\phi}=512$ and $N_{Y l m}=48$ for model HR (Boss 2001).

The three new models varied the choice for the critical disk density $\left(\rho_{c r}=10^{-13}, 10^{-12}\right.$,

or $10^{-11} \mathrm{~g} \mathrm{~cm}^{-3}$ ) below which the disk temperature was forced back to its initial value (typically $40 K$ in the outer disk). This artifice was employed in Boss (2001) and subsequent models in order to maintain a reasonably large time step when low density regions develop in the disk that are undergoing decompressional cooling.

In all three models, spiral arms and transient clumps form within 200 yrs of evolution, 
similar to the behavior with the usual (Boss 1984) pressure EOS (e.g., Boss 2001). Relatively high density clumps form, with maximum densities similar to those in a calculation with the same spatial resolution but the usual pressure EOS. Evidently using the $\gamma=5 / 3$ EOS does not alter the results in a significant way because the Boss EOS also has $\gamma=5 / 3$ for $T<100 K$, the regime where clumps form. The choice of $\rho_{c r}$ makes little difference as well, as was found by Boss (2006b) for disk instability models in binary star systems. These results suggest that the reason for differing outcomes must be sought elsewhere (see Discussion).

\section{Varied Disk Temperature Parameters}

We now present a set of four models varying several of the parameters that could affect the temperature distribution in the disk models. Table 1 summarizes the four models, which are all variations on model HR of Boss (2001). However, these models all had the same spatial resolution $\left(N_{\phi}=512\right)$ and number of terms in the spherical harmonic expansion for the gravitational potential $\left(N_{Y l m}=48\right)$ as model HR in Boss $(2001)$. The parameters that were varied included: the temperature of the thermal bath (i.e., the envelope temperature $T_{e}$ ), the critical density in the disk below which the temperature was reset to the initial disk temperature at that orbital radius (as in the previous section), the critical density in the envelope below which the gas was assumed to be at a temperature equal to the envelope temperature $T_{e}$ (for grid points at least 8 degrees above the disk midplane), and finally, whether the temperature was forced to decline monotonically (Boss 2002a) with vertical height inside the disk (mono) or not (free). The former constraint errs on the side of artificially cooling the disk by removing local temperature maxima in the vertical direction. All models started at a time of 322 yrs of evolution in model HR, and continued for at least another 17 yrs of evolution ( $\sim 1$ clump orbital period).

The results of these variations on the standard assumptions are shown in Figures 2 and 3. The variation that produced the largest deviation from the standard assumptions (model $\mathrm{H}$ in Figure 2) was relaxing the constraint on the monotonic vertical (more precisely, in the $\theta$ angle) decline of temperatures within the disk (model TZ in Figure 3), though even this model led to the formation of well-defined clumps that were no more than a factor of two less dense than in model H. Models T and TE led to evolutions that were very similar to that of model $\mathrm{H}$ and so are not shown. The models show that these three variations in the details of how the disk thermodynamics is treated in the Boss models are not particularly significant for the outcome of a disk instability, presumably because of the thermal bath assumption. 


\section{Discussion}

There are a number of possible reasons for different outcomes compared to other groups:

Spatial resolution - Clump formation is strongly enhanced as the spatial resolution in the critical azimuthal direction is increased from $N_{\phi}=64$ to 512 (Boss 2000). Boss (2005) presented a model with $N_{\phi}=1024$ and a locally refined radial grid (equivalent to a calculation with over $8 \times 10^{6}$ grid points) that implied that in the continuum limit, the outcome of a disk instability is the formation of dense, self-gravitating clumps. Cai et al. (2006a,b) calculated models with $N_{\phi}=128$, increasing $N_{\phi}$ to 512 for two models only after those models had entered a phase of evolution when nonaxisymmetry was no longer growing. Boley et al. (2006) similarly calculated models with $N_{\phi}=128$, increasing $N_{\phi}$ for some models to 512 for the earliest phase of evolution, leading to the formation of dense clumps at the intersections of spiral structures. The clumps disappeared in a fraction of an orbital period. Clumps typically last no more than an orbital period in even the highest spatial resolution models of Boss (2005). Boss (2005) thus used virtual protoplanets to allow the orbital evolution of these dense clumps to be followed further than is possible with even a high spatial resolution calculation with a fixed Eulerian grid code.

Gravitational potential solver - The Boss models use a spherical harmonic $\left(Y_{l m}(\theta, \phi)\right)$ expansion to solve Poisson's equation for the gravitational potential, with the accuracy of the resulting gravitational potential being strongly dependent on the number of terms $\left(N_{Y l m}\right)$ carried along in the expansion. As we have seen, model HR in Boss (2001) used $N_{Y l m}=48$. Boss $(2000,2001)$ found that increasing $N_{Y l m}$ led to the formation of significantly denser clumps. Boss (2005) further explored the effects of using an enhanced gravitational potential solver by replacing some of the mass in the densest regions of a clump with a point mass at the center of the relevant grid cell, finding that this led to even better defined, higher density clumps. In comparison, the IU group uses a direct solution of Poisson's equation, with a boundary potential employing terms up to $l=m=10$ (Pickett et al. 2000), implying a limited ability to depict small-scale gravitational forces in a strongly nonaxisymmetric disk. Mejía (2004) considered Fourier analysis of her disk models for $m \leq 6$, while Boley et al. (2006) considered $m \leq 63$, finding increasingly little power in modes with $m>10$, possibly consistent with the cutoff at $l=m=10$ in their boundary potential.

Artificial viscosity - Pickett \& Durisen (2007) found that the inclusion of certain artificial viscosity (AV) terms could enhance the survival of clumps formed in a disk instability, and suggested that AV could thus explain the long-lived clumps found in SPH calculations by Mayer et al. (2002, 2004, 2007). Pickett \& Durisen (2007) further noted that even calculations without any explicit AV (e.g., the Boss models) should be considered suspect, given the intrinsic numerical viscosity of any numerical code. Boss (2006b) showed that with 
a large amount of explicit AV, clump formation is suppressed, as was also found by Pickett \& Durisen (2007). However, the level of intrinsic numerical viscosity in Boss code models with $N_{\phi}=256$ appears to be equivalent to an $\alpha$-viscosity of $\sim 10^{-4}$ or smaller (Boss 2004), a level that appears to be negligible in comparison to typical explicit AV levels. Considering that the virtual protoplanet (VP) models of Boss (2005) had $N_{\phi}=256$, the continued survival of the VP for at least 30 orbital periods in these models is not likely to have been affected by the intrinsic numerical viscosity of the Boss code.

Stellar irradiation - Mejía (2004) considered the effects of stellar irradiation on the surface of the disk as a means of heating the disk surface and thereby possibly suppressing clump formation. The Boss models assume that the disk is immersed in a thermal bath appropriate for backscattering from infalling dust grains in the protostellar envelope, with an envelope temperature appropriate for a protostar that is not undergoing an FU Orionis outburst (Chick \& Cassen 1997). While the Boss models thus do not include the effects of direct irradiation by the central protostar, the dynamical evolution of a three dimensional disk leads to strongly variable vertical oscillations and structures (Mejía 2004; Boley \& Durisen 2006; Jang-Condell \& Boss 2007) that are not considered in simple theoretical models of flared accretion disks. The highly corrugated inner disk surfaces (stretching at least 29 degrees above the disk midplane; Jang-Condell \& Boss 2007) will shield the outer disk surfaces from the central protostar, eliminating this source of heating for much of the outer disk.

Radiative transfer - Boley et al. (2006) have calculated disk instability models using the flux-limited diffusion approximation (FLDA) along with a detailed treatment of the transition from the optically thick disk to the optically thin atmosphere of the disk. They suggest using a plane-parallel (one dimensional) atmosphere as a test case. Boss (2001) investigated the effects of using the FLDA instead of the standard diffusion approximation (DA) coupled with a thermal bath for low optical depths, but did not find any significant differences. Myhill \& Boss (1993) showed the results of the fully three dimensional, standard nonisothermal test case for protostellar collapse, calculated with their two independent Eddington approximation (EA) codes, finding good agreement. Whitehouse \& Bate (2006) found that their FLDA models of the standard nonisothermal collapse problem led to temperature profiles similar to those found by Myhill \& Boss (1993), though with appreciably hotter gas temperatures where the optical depth was $\sim 2 / 3$. They attribute this difference to the FLDA retarding the loss of radiation in these layers compared to the EA. Given that the choice of the flux limiter can have an effect on the outcome (Bodenheimer et al. 1990), it is unclear whether any particular implementation of the FLDA is superior to the EA. The standard Boss models have used the DA coupled with a thermal bath to force the DA models to mimic an EA calculation. 
Numerical heating - In calculations by the IU group, nonaxisymmetric perturbations tend to grow rapidly for a certain period of time and then begin to damp out (e.g., Cai et al. 2006a,b). In the Boley et al. (2006) calculations, the disk starts out with a mass of 0.07 $M_{\odot}$ and a radius of $40 \mathrm{AU}$, but then expands outward to a radius of $\sim 80 \mathrm{AU}$, leading to the formation of rings inside $20 \mathrm{AU}$ and a gravitationally stable region outside $20 \mathrm{AU}$ with spiral arms that do not fragment. The latter behavior is roughly consistent with the models by Boss (2003), who studied disks extending from $10 \mathrm{AU}$ to $30 \mathrm{AU}$, and found fragments to form at 20 AU but not at 30 AU. Similarly, Boss (2006a) studied disks extending from $100 \mathrm{AU}$ to $200 \mathrm{AU}$, and found no evidence for fragmentation. Thus on scales larger than $\sim 20$ AU, the results of Boley et al. (2006) and Boss (2003, 2006a) are in basic agreement. The disagreement arises about what happens in the inner disks. Fragmentation typically occurs at 8 to $10 \mathrm{AU}$ in the Boss models, whereas the inner disk rings do not fragment in the Boley et al. (2006) models. Boley et al. (2006) state that their inner disk is stable to ring fragmentation because of "numerical heating" at distances out to $\sim 7$ AU. This non-physical heating appears to have affected the models of Cai et al. (2006a,b) as well as those of Boley et al. (2006), making the IU results for inner disks difficult to accept.

\section{Conclusions}

While there are a number of potential reasons for the differences in disk instability models calculated by the IU group and the present author, at this time the major sources of these differences would appear to be some combination of several effects, namely spatial resolution, gravitational potential solver accuracy, and numerical heating in the inner disk of the IU models. Handling of the boundary conditions for the disk's radiative energy losses is another possibility that is still under investigation by the author, though the models presented here suggest that this may not be a dominant effect. Given the current state of knowledge, and the new results presented herein, it appears that reports of the death of the disk instability model for giant planet formation have been greatly exaggerated.

I thank Aaron Boley, Kai Cai, and Megan Pickett for working with me to understand the differences between their group's results and my results, and the referees for their remarks. This research was supported in part by NASA Planetary Geology and Geophysics grant NNG05GH30G and is contributed in part to NASA Astrobiology Institute grant NCC2-1056. The calculations were performed on the Carnegie Alpha Cluster, the purchase of which was partially supported by NSF Major Research Instrumentation grant MRI-9976645. 


\section{REFERENCES}

Bodenheimer, P., Yorke, H. W., Różyczka, M., \& Tohline, J. E. 1990, ApJ, 355, 651

Boley, A. C., \& Durisen, R. H. 2006, ApJ, 641, 534

Boley, A. C., et al. 2006, ApJ, 651, 517

Boley, A. C., Hartquist, T. W., Durisen, R. H., \& Michael, S. 2007a, ApJL, in press

—. 2007b, ApJL (Erratum), submitted

Boss, A. P. 1984, ApJ, 277, 768

—. 1989, ApJ, 346, 336

—. 1997, Science, 276, 1836

—. 2000, ApJL, 536, L101

—. 2001, ApJ, 563, 367

—. 2002a, ApJ, 567, L149

—. 2002b, ApJ, 576, 462

—. 2003, ApJ, 599, 577

—. 2004, ApJ, 616, 1265

—. 2005, ApJ, 629, 535

—. 2006a, ApJL, 637, L137

—. 2006b, ApJ, 641, 1148

-. 2006c, ApJL, 643, 501

Cai, K., et al. 2006a, ApJL, 636, L149

—. 2006b, ApJL (Erratum), 642, L173

Chick, K. M., \& Cassen, P. 1997, ApJ, 477, 398

Cox, J. P., \& Giuli, R. T. 1968, Principles of Stellar Structure (New York, Gordon and Breach)

Durisen, R. H., et al. 2007, in Protostars and Planets V, B. Reipurth, D. Jewitt, \& K. Keil, eds. (Tucson, Univ. Arizona Press), p. 607

Jang-Condell, H., \& Boss, A. P. 2007, ApJL, in press

Mayer, L, Quinn, T., Wadsley, J., \& Stadel, J. 2002, Science, 298, 1756

—. 2004, ApJ, 609, 1045 
- 2007, astro-ph/0606361

Mejía, A. C. 2004, PhD thesis, Indiana Univ.

Myhill, E. A., \& Boss, A. P. 1993, ApJS, 89, 345

Pickett, B. K., Cassen, P., Durisen, R. H., \& Link, R. 2000, ApJ, 529, 1034

Pickett, M. K., \& Durisen, R. H. 2007, ApJL, 654, L155

Whitehouse, S. C., \& Bate, M. R. 2006, MNRAS, 367, 32 
Table 1. Models with varied disk temperature parameters.

\begin{tabular}{ccccc}
\hline \hline model & $T_{e}(\mathrm{~K})$ & $\rho_{c r}($ disk $)$ & $\rho_{c r}$ (envelope) & $T(\theta)$ \\
\hline $\mathrm{H}$ & $50 \mathrm{~K}$ & $10^{-11}$ & $10^{-11}$ & mono \\
$\mathrm{T}$ & $100 \mathrm{~K}$ & $10^{-13}$ & $10^{-11}$ & mono \\
$\mathrm{TZ}$ & $50 \mathrm{~K}$ & $10^{-13}$ & $10^{-11}$ & free \\
$\mathrm{TE}$ & $50 \mathrm{~K}$ & $10^{-13}$ & $10^{-13}$ & mono \\
\hline
\end{tabular}




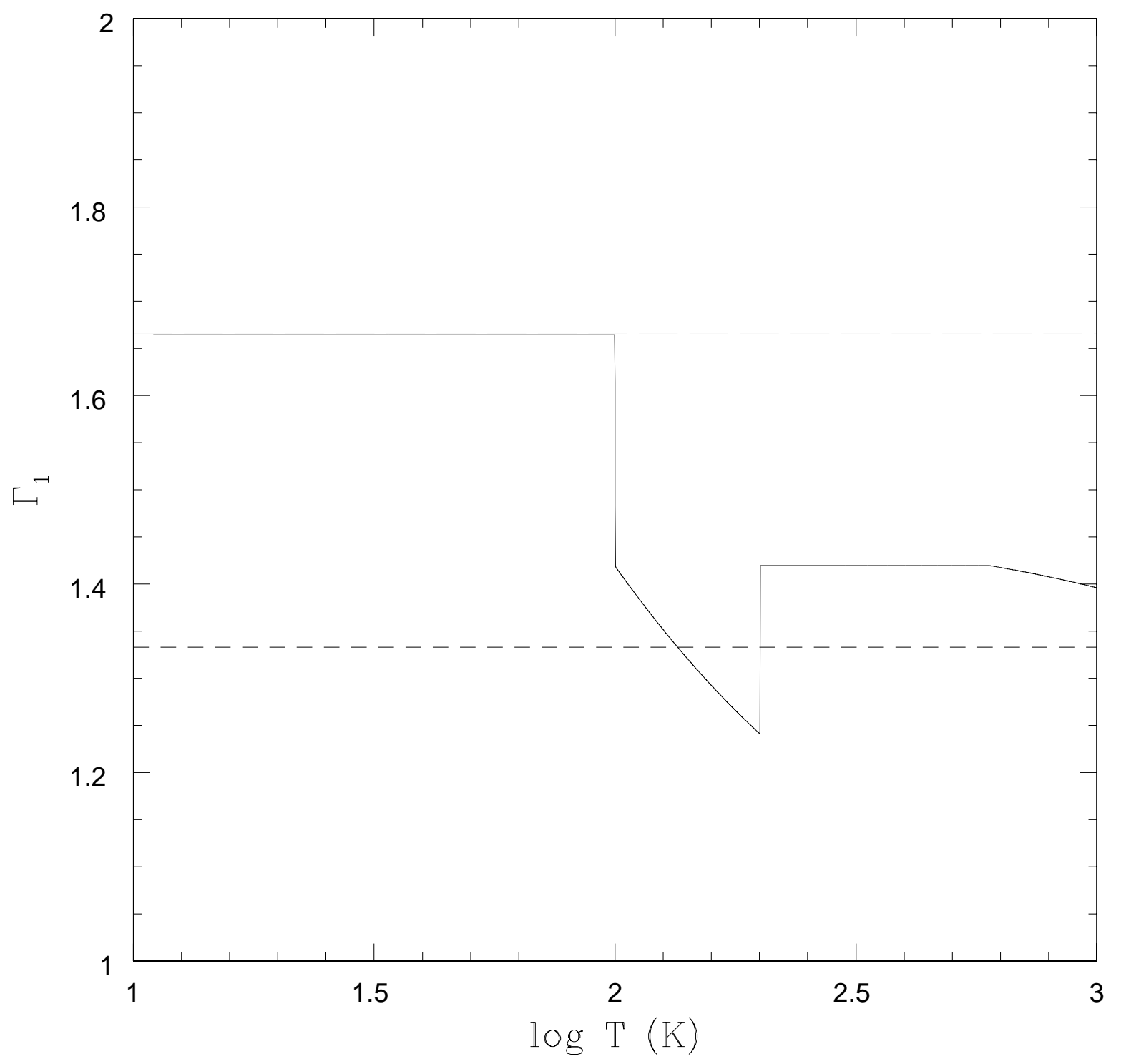

Fig. 1.- Adiabatic exponent $\Gamma_{1}=\gamma$ used in Boss (2001) and in all subsequent Boss disk instability models (solid line). The short-dashed line shows $\gamma=4 / 3$, while the long-dashed line shows $\gamma=5 / 3$, as used by Cai et al. (2006a,b) and Boley et al. (2006). 


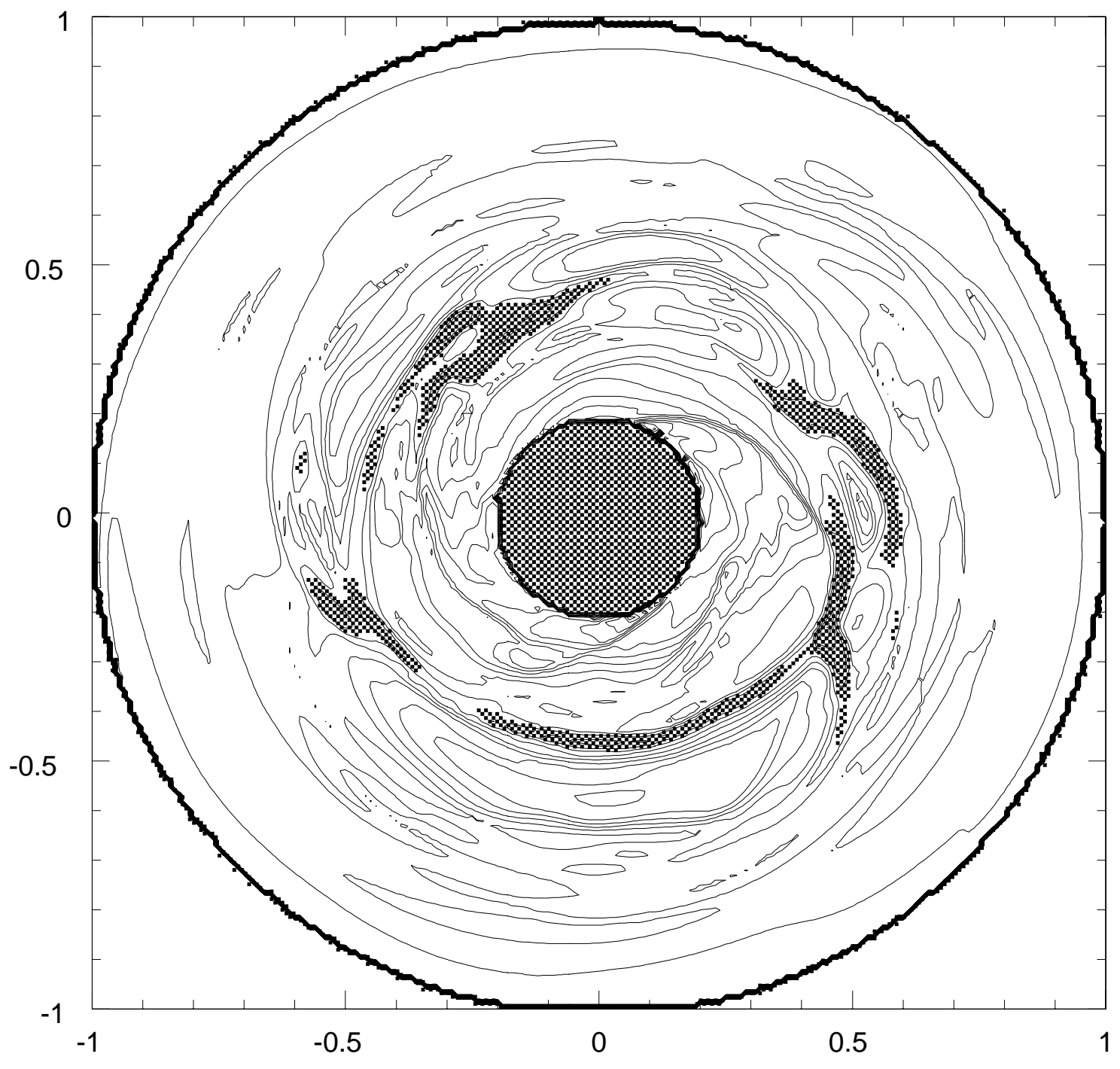

Fig. 2.- Equatorial density contours for model $\mathrm{H}$ after 339 yrs of evolution. The disk has an outer radius of $20 \mathrm{AU}$ and an inner radius of $4 \mathrm{AU}$. Hashed regions denote clumps and spiral arms with densities higher than $10^{-10} \mathrm{~g} \mathrm{~cm}^{-3}$. Density contours represent factors of two change in density. 


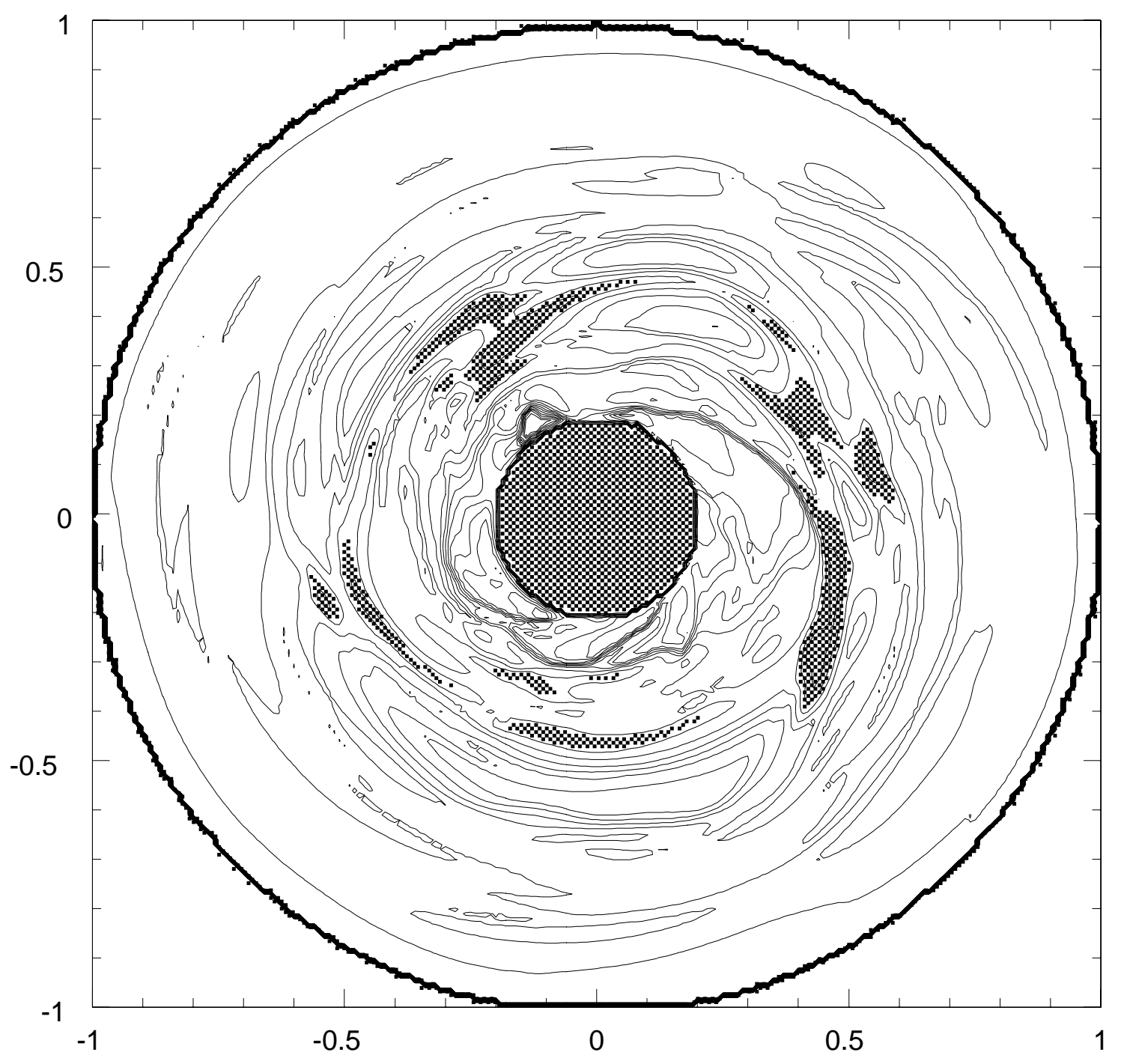

Fig. 3.- Same as Figure 2, but for model TZ. 